Article

\title{
Campylobacter jejuni Developed the Resistance to Bacteriophage CP39 by Phase Variable Expression of 06875 Encoding the CGPTase
}

\author{
Yuanyue Tang ${ }^{1,2,3,+} \mathbb{D}$, Jie Li ${ }^{1,2,3,+}$, Yuexuan Wang ${ }^{1,2,3}$, Zhaojun Song ${ }^{4}$, Hangning Ying ${ }^{1,2,3}$, Linghua Kong ${ }^{5}$, \\ Xin'an Jiao ${ }^{1,2,3}$ (D) and Jinlin Huang 1,2,3,*
}

1 Key Laboratory of Prevention and Control of Biological Hazard Factors (Animal Origin) for Agri-Food Safety and Quality, Ministry of Agriculture of China, Yangzhou University, Wenhui East Road 48, Yangzhou 225009, China; tangyy@yzu.edu.cn (Y.T.); lijie0360@163.com (J.L.); wyx20010524@163.com (Y.W.); yhangning@163.com (H.Y.); jiao@yzu.edu.cn (X.J.)

2 Jiangsu Key Laboratory of Zoonosis/Jiangsu Co-Innovation Center for Prevention and Control of Important Animal Infectious Diseases and Zoonoses, Yangzhou University, Wenhui East Road 48, Yangzhou 225009, China

3 Joint International Research Laboratory of Agriculture and Agri-Product Safety, Yangzhou University, Wenhui East Road 48, Yangzhou 225009, China

4 The Fourth Affiliated Hospital, Zhejiang University School of Medicine, No.1 Shang Cheng Avenue, Yiwu 322000, China; 8619003@zju.edu.cn

5 Department of Quality and Safety Control, Heyi Food Co., Ltd., Zaozhuang 277500, China; klh6668@126.com

* Correspondence: jinlin@yzu.edu.cn

+ These authors contributed equally to this work.

check for updates

Citation: Tang, Y.; Li, J.; Wang, Y.; Song, Z.; Ying, H.; Kong, L.; Jiao, X.; Huang, J. Campylobacter jejuni Developed the Resistance to Bacteriophage CP39 by Phase Variable Expression of 06875 Encoding the CGPTase. Viruses 2022, 14, 485. https://doi.org/10.3390/ v14030485

Academic Editor: Alain Dublanchet

Received: 26 January 2022

Accepted: 25 February 2022

Published: 26 February 2022

Publisher's Note: MDPI stays neutral with regard to jurisdictional claims in published maps and institutional affiliations.

Copyright: (C) 2022 by the authors. Licensee MDPI, Basel, Switzerland. This article is an open access article distributed under the terms and conditions of the Creative Commons Attribution (CC BY) license (https:// creativecommons.org/licenses/by/ $4.0 /)$.

\begin{abstract}
Bacteriophage (phage) is regarded as an antimicrobial alternative for Campylobacter in food production. However, the development of phage resistance to the host is a main concern for the phage application. This study characterized the phage CP39 and investigated the phage resistance of CP39 in Campylobacter jejuni NCTC12662. We determined that phage CP39 belonged to the Myoviridae family by the WGS and phylogenetic analysis. Phage CP39 was confirmed as a capsular polysaccharide (CPS)-dependent phage by primary C. jejuni phage typing. It was further confirmed that the phage could not be adsorbed by the acapsular mutant $\triangle k p s M$ but showed the same lytic ability in both the wild-type strain NCTC 12662 and the $\triangle m o t A$ mutant lacking motile flagella filaments. We further determined that the 06875 gene encoding CDP-glycerol:poly (glycerophosphate) glycerophosphotransferase (CGPTase) in the CPS loci was related to phage CP39 adsorption by SNP analysis and observed a rapid development of phage resistance in NCTC 12662 during the phage infection. Furthermore, we observed a high mutation frequency of 06875 (32\%), which randomly occurred in nine different sites in the gene according to colony PCR sequencing. The mutation of the 06875 gene could cause the phase variable expression of non-functional protein and allow the bacteria against the phage infection by modifying the CPS. Our study confirmed the 06875 gene responsible for the CPS-phage adsorption for the first time and demonstrated the phase variable expression as a main mechanism for the bacteria to defend phage CP39. Our study provided knowledge for the evolutionary adaption of bacteria against the bacteriophage, which could add more information to understand the phage resistance mechanism before applying in the industry.
\end{abstract}

Keywords: bacteriophage resistance; Campylobacter jejuni; CPS; phage resistance

\section{Introduction}

Bacteriophages (or phages) play an essential part in shaping the bacterial ecosystem by the lytic process, while bacteria usually react constantly to promote a defense action during phage infection [1]. For all bacteriophages, the approach to infect bacteria starts 
by recognizing the specific receptor and attaching the phage particle to the bacterial surface via a receptor-binding protein (RBP) on the tail. By contracting the tail sheath, the phage contracts the tail to penetrate the cell membrane by lyases, injecting phage DNA into the cell, and using the host materials for proliferation. However, the constant phage exposure promotes bacteria to develop mechanisms specially against phage infection. The phage resistance mechanisms can either be the external prevention of phage to inject DNA by blocking or modifying phage binding receptors on the cell membrane or the internal degradation of the foreign phage genomic DNA, including the restriction-modification systems, abortive infection systems, and the CRISPR-cas interference [2]. For bacteria, the initial defense strategy is to modify the phage receptor and prevent phage absorption, which is also the most common bacterial resistance strategy during phage-host interaction [2]. Phage receptors in Gram-negative bacteria include the outer membrane proteins, lipopolysaccharides (LPS), capsular polysaccharides (CPS), pili, and flagella, which unique surface structures could ensure phage binding to the correct host [3].

Campylobacter jejuni is the commensal bacteria in poultry, and it is also one of the most common foodborne pathogens that cause bacterial gastroenteritis in humans worldwide. C. jejuni are transmitted to humans by eating uncooked meat products or directly contacting livestock during food production. Humans can be infected by eating poultry contaminated with $C$. jejuni, and threaten public health $[4,5]$. Bacteriophage is one of the alternatives against $C$. jejuni contamination in food production [6]. C. jejuni phages are known to co-exist with their host and could commonly be isolated from chicken feces and poultry-related production environments [7].

Most C. jejuni phages belong to the Myoviridae family, which are divided to group I (320-425 kbp), group II (175-183 kbp), and group III (131-135 kbp) according to genome size. C. jejuni phage receptors include flagella and capsular polysaccharides (CPS), while the loss of flagella motility or CPS can lead to the development of phage resistance $[8,9]$. A previous study has demonstrated that the flagella-dependent phage F341 attached to the flagella and required the motility for phage binding to $C$. jejuni, while the absence of flagella motility would prevent the phage attachment [10]. C. jejuni could defend CPSdependent phage by phase variable expression of the phage receptor in the CPS loci. The O-methyl phosporamidate $(\mathrm{MeOPN})$ transferase encoded by cj1421 is involved in CPS biosynthesis, which is also responsible for the F366 phage binding in C. jejuni NCTC 11168 . The frameshifting expression of $c j 1421$ due to the addition of guanine in poly G tract could allow the host to resist against the phage F366 [11]. In addition, it has been shown that the infection of CPS-dependent phages was more than the presence of $\mathrm{MeOPN}$, indicating the diverse mechanisms of the initial phage interaction with C. jejuni [12].

C. jejuni NCTC12662 is widely used for phage isolation due to its sensitivity to many phages. The MeOPN encoded by the 06875 gene in NCTC 12662 served as a phage receptor for phage F207. The shift-off of this gene by poly $\mathrm{G}$ phase variation could help the bacteria rapidly develop phage resistance [12]. This study isolated a phage CP39 from sewage in the retail market of Yangzhou, China, using C. jejuni NCTC12662 as the host. After the phenotypic and genotypic characterization of phage CP39, we investigated the development of resistance mechanisms in NCTC 12662 during the phage infection. Our study demonstrated the diverse resistant mechanisms in C. jejuni during the phagehost interaction.

\section{Materials and Methods}

\subsection{Bacterial Strains, Bacteriophage, Plasmids and Culture Conditions}

All bacterial strains and plasmids used in this study are listed in Table S1. Briefly, C. jejuni were grown on Mueller-Hinton (MH) agar (BD Biosciences, Sparks, NV, USA) supplemented with $5 \%$ sheep blood or Campylobacter blood-free selective agar containing charcoal cefoperazone deoxycholate (CCDA) (Oxoid, Basingstoke, UK), and incubated at $42{ }^{\circ} \mathrm{C}$ under microaerobic conditions $\left(85 \% \mathrm{~N}_{2}, 10 \% \mathrm{CO}_{2}, 5 \% \mathrm{O}_{2}\right)$. E. coli $\mathrm{DH} 5 \alpha$ strains were inoculated on Luria-Bertani (LB) agar (Sangon Biotech, Shanghai, China) at $37^{\circ} \mathrm{C}$. The 
pMD-20T was used as a suicide plasmid in the construction of mutant strains. The pRK2013 is a helper plasmid for triparental mating conjugation, while pUOA18 and pRY107 are C. jejuni shuttle vectors. C. jejuni phage CP39 was originally isolated from sewage in a retail market from Yangzhou, China and is propagated on C. jejuni NCTC12662. Phages were stored in SM buffer (0.05 M Tris- $\mathrm{HCl}$, pH 7.5 with $5.8 \mathrm{~g} \mathrm{NaCl}, 2.0 \mathrm{~g} \mathrm{MgSO} \cdot 7 \mathrm{H}_{2} \mathrm{O}$, and $5 \mathrm{~mL}$ gelatin, $2 \% w / v$ solution) at $4{ }^{\circ} \mathrm{C}$.

The phage propagation was performed as previously described with minor modification [12]. Briefly, the host strain C. jejuni NCTC 12662 was grown overnight on CCDA plate and harvested by BHI broth (BD Biosciences, Sparks, NV, USA) supplemented with $10 \mathrm{mM} \mathrm{MgSO}_{4}$ and one $\mathrm{mM} \mathrm{CaCl}_{2}(\mathrm{cBHI})$ before adjusting the suspension to $\mathrm{OD}_{600}$ of 0.35 . The suspension was incubated at $37^{\circ} \mathrm{C}$ for $4 \mathrm{~h}$ under microaerobic conditions and mixed with phage CP39 at a multiplicity of infection (MOI) of 0.01 . The mixture was incubated at $37^{\circ} \mathrm{C}$ for $15 \mathrm{~min}$ for phage absorption, and with $5 \mathrm{~mL}$ NZCYM (Sangon Biotech, Shanghai, China) and $0.6 \%$ agar before pouring onto the NZCYM $1.2 \%$ agar plate and incubating at $42{ }^{\circ} \mathrm{C}$ for $4 \mathrm{~h}$ under microaerobic condition. The overlay agar was collected, vortexed with $5 \mathrm{~mL} \mathrm{SM}$ buffer, and centrifuged at $8500 \mathrm{rpm}, 4^{\circ} \mathrm{C}$ for $10 \mathrm{~min}$. The supernatant was filtered through a sterile $0.22-\mu \mathrm{m}$ membrane filter.

\subsection{Bacteriophage Titration}

Bacteriophage titration was performed as described previously [13]. C. jejuni was inoculated on $\mathrm{MH}$ agar with $5 \%$ sheep blood and incubated at $42{ }^{\circ} \mathrm{C}$ overnight under microaerobic conditions before suspending in cBHI broth. The bacterial culture was adjusted to $\mathrm{OD}_{600}$ of 0.35 and incubated at $42^{\circ} \mathrm{C}$ for $4 \mathrm{~h}$ under microaerobic conditions. The bacteriophage titer was evaluated by the double-layer agar plate assay, whereby $100 \mu \mathrm{L}$ recipient strain with $\mathrm{OD}_{600}$ of 0.5 was mixed with $100 \mu \mathrm{L}$ serially diluted phage stock in $5 \mathrm{~mL}$ soft agar and poured on a NZCYM agar plate for the count of phage plaques. For the spot assay, phage stock was serially diluted in SM buffer before $10 \mu \mathrm{L}$ phage stock was spotted on the soft agar plate with $100 \mu \mathrm{L}$ recipient strain NCTC 12662 with $\mathrm{OD}_{600}$ of 0.5 and incubated at $42{ }^{\circ} \mathrm{C}$ for $24 \mathrm{~h}$ under microaerobic conditions.

\subsection{Transmission Electron Microscopy (TEM) of Phage CP39}

TEM analysis was performed as previously described with modifications [14]. One hundred $\mu \mathrm{L}$ phage stock was dropped onto a mica sheet $(\sim 3 \times 3 \mathrm{~mm}$ size $)$ and absorbed for $10 \mathrm{~min}$. The sample was stained with $2 \%(w / v)$ uranyl acetate and virtualized with the transmission electron microscope (Tecnai 12; Philips; The Netherlands).

\subsection{Phage Genome Sequencing and Phylogenetic Analysis}

Phage DNA was extracted with the phage titer of $10^{8}-10^{9} \mathrm{PFU} / \mathrm{mL}$ using the ABigen $\lambda$ Phage DNA Purification kit (ABigen Corporation, Beijing, China). The phage genome was sequenced by Hiseq sequencer (Illumina Inc., San Diego, CA, USA) and assembled by Newbler 2.9 (Roche Diagnostics, Branford, CT, USA). Phage CP39 genome was submitted to European Nucleotide Archive (ENA) database with the accession number MH107028. The phylogenetic analysis of phage CP39 was based on the major capsid protein by CLC genenomic workbench. All reference strains for the phylogenetic analysis are listed in Table S2.

\subsection{Construction of C. jejuni Mutant and Complemented Strains}

$\Delta m o t A, \Delta k p s M, \Delta f l a A B, \Delta 06810$, and $\Delta 06875$ mutants were constructed in C. jejuni NCTC12662 by replacing target genes with aphA encoding the kanamycin-resistant cassette or cat encoding chloramphenicol resistance as previously described (Table S1) [14]. In brief, the up and down flanking regions of $\operatorname{mot} A, k p s M, f l a A B, 06810,06875$, and kanamycin resistant gene aph were amplified by PCR using the primers list in Table S3. PCR fragments and pMD-20T were ligated together by a One Step Cloning Kit (Vazyme, Nanjing, China). The recombinant plasmid was electroporated into C. jejuni competent cell, inoculated on 
CCDA agar with $50 \mu \mathrm{g} / \mathrm{mL}$ kanamycin, and incubated at $42^{\circ} \mathrm{C}$ for $24 \mathrm{~h}$ under microaerobic conditions. All mutants were confirmed by PCR sequencing, and the detailed information is listed in Table S1.

The 06875 complement strain was constructed by amplifying the 06875 gene by primers 06875-pUOA18-F/R and ligating downstream of the promoter Pmetk in pUOA18. The pUOA18-pmetK-06875 was transferred to the recipient strain NCTC12662 $\triangle 06875$ by triparental mating with the help of plasmid pRK2013. The mixture was inoculated on $\mathrm{MH}$ agar with chloramphenicol $(20 \mu \mathrm{g} / \mathrm{mL})$, polymyxin B $(6.7 \mu \mathrm{g} / \mathrm{mL})$, rifampicin $(10 \mu \mathrm{g} / \mathrm{mL})$, and trimethoprim $(5 \mu \mathrm{g} / \mathrm{mL})$, and incubated at $42{ }^{\circ} \mathrm{C}$ for $24 \mathrm{~h}$ under microaerobic conditions [15]. The complement strain was confirmed by PCR sequencing and named as $\Delta$ 06875:: $\mathrm{P}_{06875}$ (Table S1).

The point mutation of 06875 gene was constructed by amplifying the gene with primers 559A-up-F/R and 559A-down-F/R containing mutant basepairs and ligating downstream of the promoter Pmetk in pUOA18. The pUOA18-pmetK-06875-559A was mobilized into 06875 mutant strain by triparental mating with the help of the pRK2013, and inoculated on $\mathrm{MH}$ agar with chloramphenicol $(20 \mu \mathrm{g} / \mathrm{mL})$, polymyxin B $(6.7 \mu \mathrm{g} / \mathrm{mL})$, rifampicin $(10 \mu \mathrm{g} / \mathrm{mL})$, and trimethoprim $(5 \mu \mathrm{g} / \mathrm{mL})$. The successful mutant was confirmed by sequencing and the mutant was named as 06875-492delT.

\subsection{Bacteriophage Sensitivity Assay}

C. jejuni NCTC12662, $\Delta$ mot $A, \Delta k p s M, \Delta f l a A B, \Delta 06810, \Delta 06875, \Delta 06875: P_{06875}$, and 06875-492delT sensitivity to phage CP39 was evaluated by the double-layer agar plate assay as described previously [7]. Briefly, wild-type C. jejuni NCTC12662 or its mutant strains with the $\mathrm{OD}_{600}$ of 0.5 was mixed with soft agar and poured on NZCYM agar plate. After solidification, $10 \mu \mathrm{L}$ phage stock was spotted on the bacterial lawns and incubated at $42{ }^{\circ} \mathrm{C}$ for $24 \mathrm{~h}$ under microaerobic conditions. The sensitivity of phage CP39 was evaluated by the plaque formation $(\mathrm{PFU} / \mathrm{mL}$ ) on the soft agar.

\subsection{Bacterial Growth Curve}

Overnight cultures of $C$. jejuni NCTC12662, $\Delta$ mot $A$ and $\Delta k p s M$ mutant strain were suspended and diluted in $\mathrm{MH}$ broth with $\mathrm{OD}_{600}$ value of 0.07 . Ten $\mathrm{mL}$ of the cultural suspension was incubated at $42{ }^{\circ} \mathrm{C}$ and $100 \mathrm{rpm}$ under microaerobic conditions. The $\mathrm{OD}_{600}$ value of the C. jejuni broth culture was measured every $4 \mathrm{~h}$ until $24 \mathrm{~h}$. Each experiment was performed in triplicate.

\subsection{Bacteriophage Adsorption Assay}

A bacteriophage adsorption assay was performed as previously described [16]. Briefly, phage CP39 was mixed with C. jejuni NCTC12662, $\Delta m o t A$, and $\Delta k p s M$ in cBHI broth with an MOI of 0.01 , respectively. After incubation at $42{ }^{\circ} \mathrm{C}$ with $100 \mathrm{rpm}$ for $90 \mathrm{~min}$, the mixture was centrifuged at $8500 \mathrm{rpm}$ for $10 \mathrm{~min}$, then filtered through an $0.22 \mu \mathrm{m}$ pore diameter membrane filter. The adsorption level was determined by measuring bacteriophage titration. Each experiment was performed in triplicate.

\subsection{Isolation of Phage-Resistant Variants}

A hundred $\mu \mathrm{L}$ of CP39 phage suspension with $10^{6} \mathrm{PFU} / \mathrm{mL}$ were inoculated in $5 \mathrm{~mL}$ NZCYM with $0.6 \%$ of agar and poured onto the NZCYM agar plate to create a phage lawn. C. jejuni NCTC12662 in CBHI broth was adjusted to $\mathrm{OD}_{600}$ of 0.35 and incubated at $42{ }^{\circ} \mathrm{C}$ for $4 \mathrm{~h}$ under microaerobic conditions. Subsequently, C. jejuni was serially diluted from $10^{-1}$ to $10^{-4}$ in SM buffer. Ten $\mu \mathrm{L}$ suspension was spotted on the phage lawn and incubated under microaerobic conditions until colonies were produced on the plaques. Single colonies were tested for resistance against CP39 by plaque assay. The suspicious resistant colonies were re-streaked five times on $\mathrm{MH}$ agar plates, and the resistance of colonies against CP39 was tested after each time by plaque assay. After being re-streaked five times on $\mathrm{MH}$ agar, the single colony conferring phage resistance was regarded as $C$. jejuni strain resistant 
to phage CP39. Four isolates with resistance to CP39 were randomly selected for wholegenome sequencing and named as 12662_CP39R1, 12662_CP39R2, 12662_CP39R3, and 12662_CP39R4.

\subsection{Bacterial Genome Sequencing and Analysis}

Genomic DNA was extracted from 12662_CP39R1, 12662_CP39R2, 12662_CP39R3, and 12662_CP39R4 using TIANamp Bacteria DNA Kit (Tiangen, Beijing, China) and sequenced by Illumina Hiseq 2500. Reads were assembled to contigs by SPAdes 3.12. SNPs were analyzed by mauve and mapped to the NCTC12662 reference genome with accession number NZ_CP019965 in the NCBI database. Sequences of four phage resistant isolates were uploaded to the NCBI database with the accession number PRJNA798540. Genes with SNPs from the four phage resistant isolates are listed in Table S4.

\subsection{Phage-Bacteria Co-Cultivation and the One-Step Phage Growth Curve}

Phage-resistant populations were generated via co-culture of phage and C. jejuni.

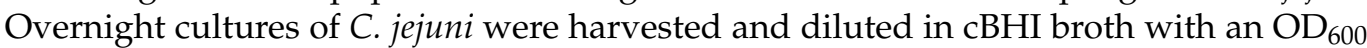
value of 0.07 . CP39 was added at MOI of 0.01 , and co-culture was incubated at $42{ }^{\circ} \mathrm{C}$ $100 \mathrm{rpm}$. The $\mathrm{OD}_{600}$ value was measured at each hour until $6 \mathrm{~h}$ and each $6 \mathrm{~h}$ until $36 \mathrm{~h}$. The one-step phage growth was also performed during the phage-host co-cultivation. At each time point, $500 \mu \mathrm{L}$ samples were centrifuged at $8500 \mathrm{rpm}$, and supernatants were filtered through a $0.22-\mu \mathrm{m}$ diameter pore membrane filter, and the concentration of phage was evaluated by PFU/mL.

\subsection{The Mutation of 06875 in NCTC12662 during Phage Infection}

After 36 h co-cultivation of phage CP39 and C. jejuni NCTC12662, the mixtures were serially diluted to $10^{-4}$ in PBS, and $100 \mu \mathrm{L}$ of each dilution was spread on the CCDA plate and incubated at $42{ }^{\circ} \mathrm{C}$ for $24 \mathrm{~h}$ under microaerobic conditions. One hundred colonies were selected for the PCR amplification of the 06875 with primers 06875 -outer-F and 06875-outer-R (Table S3). The PCR products were sequenced by Sangon Biotech and analyzed for mutations of the 06875 gene by NCBI BLAST. The mutation rate of 06875 was calculated as the number of 06875 mutants divided by the total sequenced colonies.

\section{Results}

\subsection{Identification and Characterization of Phage CP39}

Phage CP39 was isolated from the sewage of a retail market in Yangzhou, China. The phage formed circulated and transparent plaques with 1-2 mm (Figure 1A). TEM showed that phage CP39 belonged to the Myoviridae family with a head size of approximately $80 \mathrm{~nm}$ and connected to a terminal bleb at the distal ends of their tails (Figure 1B). Genome sequencing showed that the whole genome size of phage CP39 was 130,715 bp containing 168 ORF and three tRNAs. The phylogenetic analysis revealed that phage CP39 was clustered into Fletchervirus phages as reference strain NCTC12673 and was closely related to the phage vB_CjeM_los1, indicating phage CP39 as a CPS-dependent phage [17].

\subsection{The Primary Determination of CP39 Phage as a CPS-Dependent Phage}

According to the previous study, we primarily investigated the phage type and its receptor based on the genomic analysis [7]. We constructed NCTC12662 $\triangle$ motA mutant without motile flagella filaments and an acapsular kpsM mutant NCTC12662 $\Delta k p s M$ for phage typing. Both $\Delta m o t A$ and $\Delta k p s M$ mutants showed not significant growth differences from the wild type (WT) NCTC12662 (Figure S1). The receptor type of CP39 was determined by evaluating plaques formation of CP39 on the soft agar plate containing wild type, $\Delta$ mot $A$ or $\Delta k p s M$ mutant. Our results demonstrated that CP39 could form a clear plaque on wild type (Figure $2 \mathrm{~A}$ ) and $\Delta \operatorname{mot} A$ mutant (Figure $2 \mathrm{~B}$ ), while no plaque was observed on $\triangle k p s M$ (Figure 2C), indicating that the absence of CPS in the recipient strain could influence the phage adsorption. We also investigated the adsorption of phage in liquid broth and 
observed a $2 \mathrm{LogPFU} / \mathrm{mL}$ decrease of free phage in wild type and $\Delta$ mot $A$ mutant, while the free phage in $\triangle k p s M$ mutant remained stable (Figure 2D). In addition, we also constructed the mutant $\triangle$ fla $A B$ lacking the flagellar filaments and confirmed that the phage infection did not involve the flagellar (Figure S2A). The above result demonstrated that phage CP39 was a CPS-dependent phage.

A

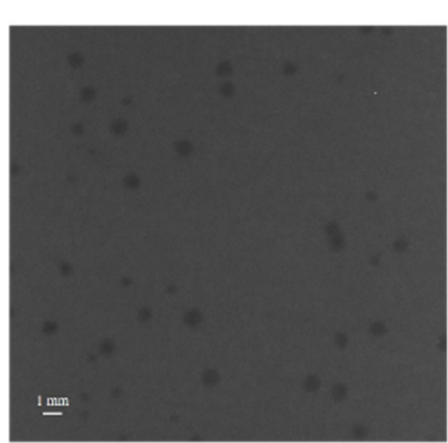

B

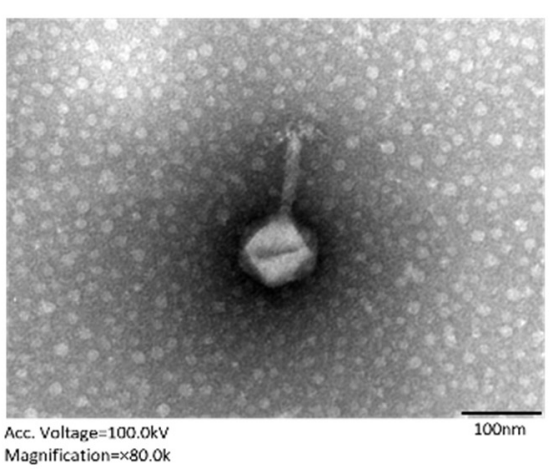

$\mathrm{C}$

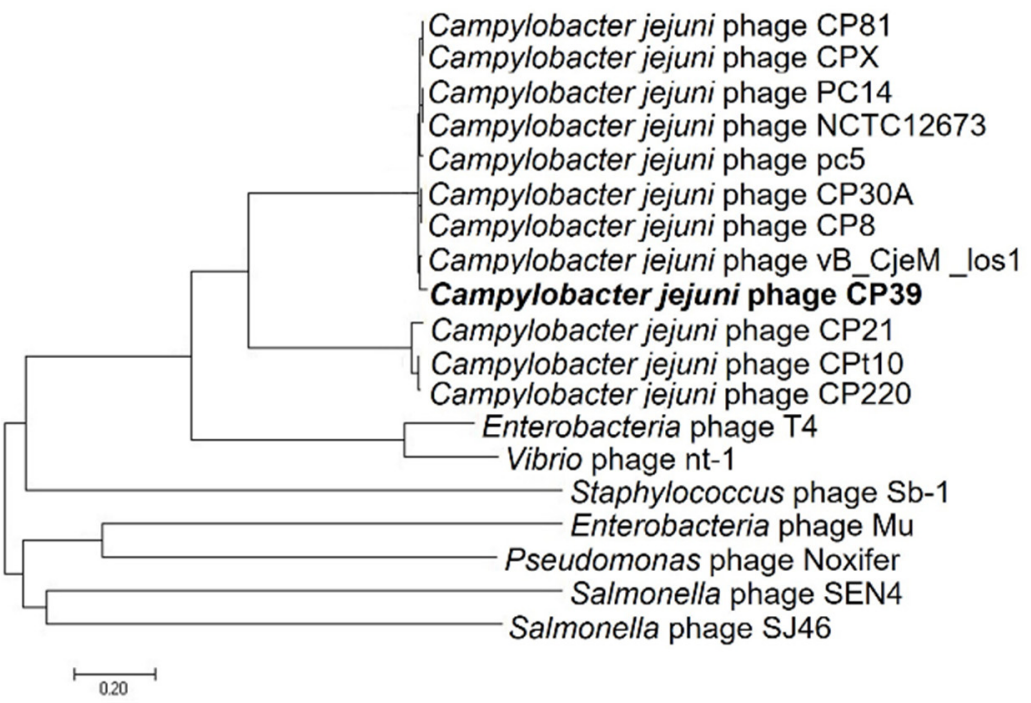

Figure 1. Identification and characterization of Campylobacter jejuni phage CP39. (A) The plaque morphology of C. jejuni phage CP39. (B) transmission electron microscopy (TEM) image of phage CP39 indicating the phage belongs to Myoviridae family. (C) The phylogenetic relationship of phage CP39. Additional information of genomes was listed in Table S2.

Previous studies have reported the $\mathrm{MeOPN}$-transferase is responsible for the infection of CPS-dependent phage in C. jejuni $[12,18,19]$. Therefore, we constructed mutant of the 06810 gene encoding the MeOPN-transferase in NCTC12662 and conducted the phage sensitivity assay to investigate if $\mathrm{MeOPN}$-transferase was responsible for the infection of phage CP39. As a result, we observed a clear plaque on the soft agar plate with $\Delta 06810$ mutate as the recipient strain (Figure 2E), indicating that the receptor of phage CP39 was not dependent on the 06810 gene, but might relate to other genes located in the CPS loci.

\subsection{The 06875 Gene Located in CPS Cluster of NCTC12662 Is Responsible for the Phage CP39 Infection}

We obtained the phage-resistant isolate by spotting serially diluted NCTC12662 strain on soft agar plate containing $10^{6} \mathrm{PFU}$ phage $\mathrm{CP} 39$, and isolated the colonies occurred on the phage lawn. Four phage-resistance isolates were randomly selected and the whole genome sequenced, including 12662_CP39R1, 12662_CP39R2, 12662_CP39R3 12662_CP39R4. After Mauve analysis, we identified several SNPs, including the B2K12_RS06420 (12662_CP39R1) encoding a motility accessory factor, B2K12_RS06335 (12662_CP39R1) and B2K12_RS06385 (12662_CP39R4) encoding flagellin modification protein PseD, B2K12_RS03815 (12662_CP39R2) encoding a hypothetical protein, B2K12_RS06875 (12662_CP39R3) encoding CDP-glycerolglycerophosphate glycerophosphotransferase (CGPTase) in the CPS loci, and B2K12_RS06565 (12662_CP39R4) encoding a nucleotidyltransferase (Table S4). The SNP that occurred 
in 06875 was one deletion of thymine $(\mathrm{T})$ in a poly $\mathrm{T}$ tract with eight Ts in NCTC12662 $(1,355,914 \sim 1,355,921 \mathrm{bp})$.

A

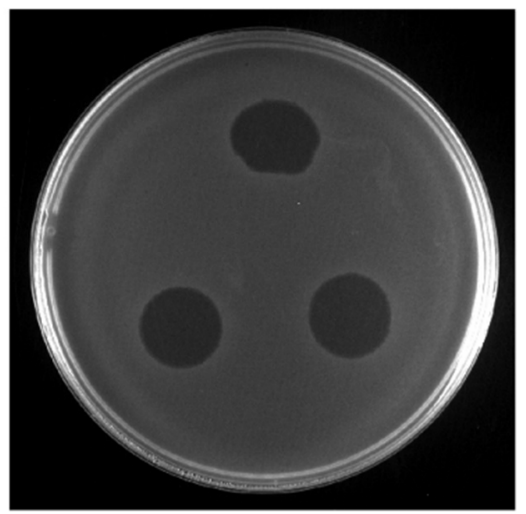

$\mathrm{WT}+\mathrm{CP} 39$
B

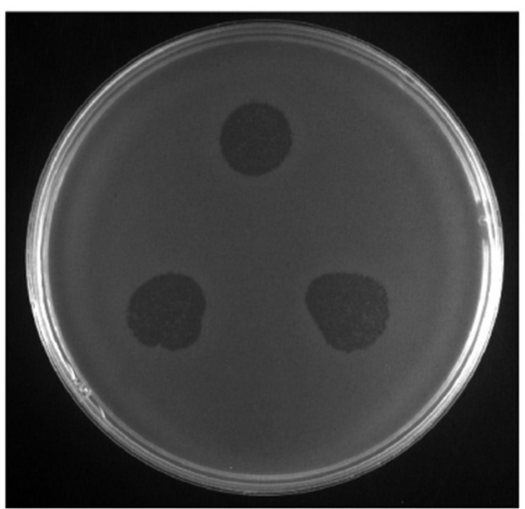

$\Delta m o t A+\mathrm{CP} 39$
C

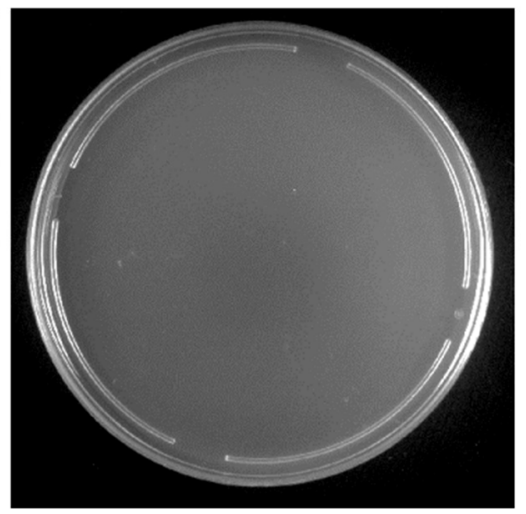

$\Delta k p s M+\mathrm{CP} 39$

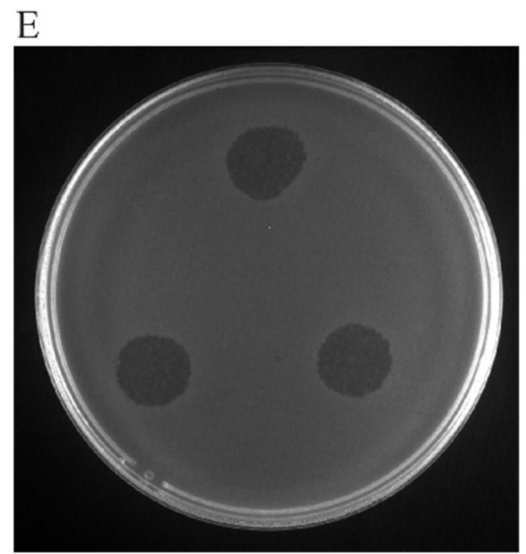

$406810+\mathrm{CP} 39$

D

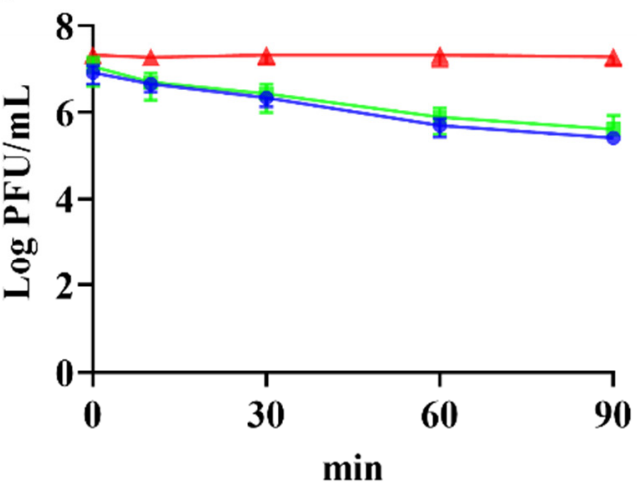

- WT+CP39

$-\Delta \operatorname{mot} A+\mathrm{CP} 39$

$\leftarrow \Delta$ kpsM+CP39

90

Figure 2. Identification of phage CP39 receptor type. (A) Sensitivity of NCTC12662 strain to CP39. (B) Sensitivity of $m o t A$ mutant strain to CP39. (C) Sensitivity of kpsM mutant strain to CP39. (D) The adsorption assay of CP39 to NCTC12662, mot $A$ and $k p s M$ mutant strain. mot $A$ and $k p s M$ mutant strains were infected with CP39 for $100 \mathrm{~min}$ at an MOI of 0.01 . The same culture NCTC12662 served as the control. (E) Sensitivity of $\Delta 06810$ mutant to CP39.

The phage resistance caused by the mutation of 06875 was further confirmed by the deletion mutant $\Delta 06875$ and its complement, $\triangle 06875:: \mathrm{P}_{06875}$. Both WT and complement strain $\Delta 06875:: \mathrm{P}_{06875}$ were sensitive to phage CP39 (Figure 3B,D), while the $\Delta 06875$ mutant showed resistance to the phage infection (Figure $3 \mathrm{C}$ ). In addition, the adsorption assay also showed that the 06875 complementary strain could partially recover the phage infection compared to the $\Delta 06875$ mutant (Figure 3D). The above results indicated that the 06875 gene was responsible for the infection of phage CP39, while the truncated expression of 06875 could cause bacterial resistance to the phage. 
A

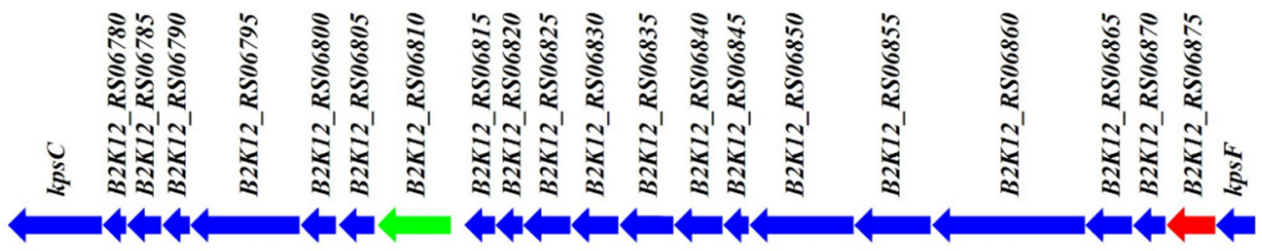

B

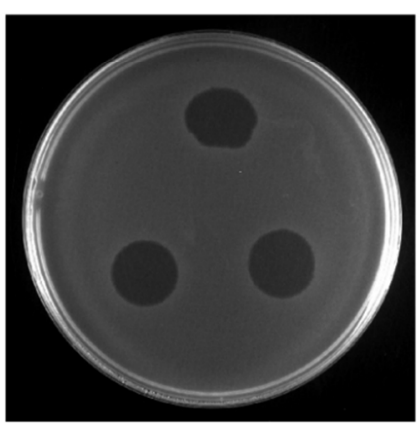

WT+CP39
$\mathrm{C}$

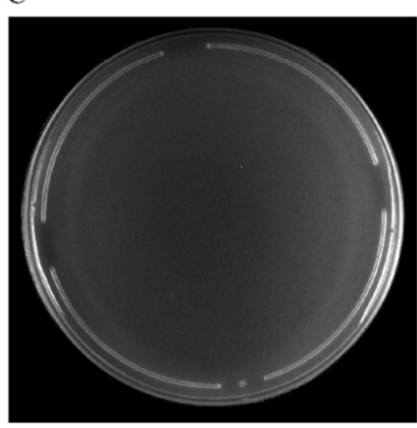

$\triangle 06875+\mathrm{CP} 39$
$\mathrm{D}$

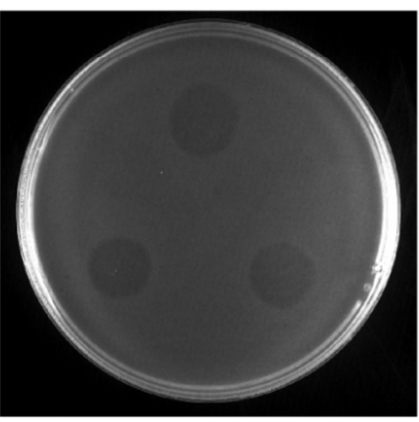

$\triangle 06875: \because \mathrm{P}_{06875}+\mathrm{CP} 39$

Figure 3. The 06875 gene in CPS loci is responsible for the CP39 resistance in C. jejuni NCTC12662. (A) Schematic diagram of CPS loci from C. jejuni NCTC12662. B2K12_RS06810 (green arrow) encodes the MeOPN-GalfNAc transferase, which the modification of MeOPN is known to be responsible for C. jejuni phage resistance. B2K12_RS06875 (red arrow) is annotated as CDP-glycerolglycerophosphate glycerophosphotransferase, which related to the phage CP39 resistance in this study. The phage sensitivity assay confirmed the 06875 gene was responsible for infection of phage CP39, which wild type strain NCTC 12662 (WT) (B) $\Delta 06875$ mutant (C) and its complementary strain $\Delta 06875:: \mathrm{P}_{06875}$ (D) was infected by CP39 on soft agar plate.

\subsection{High Mutation Frequency of 06875 Can Benefit the Bacteria to Resist Phage CP39}

The development of CP39 phage resistance in C. jejuni NCTC12662 was evaluated by the co-culture assay in $\mathrm{CBHI}$ broth. The growth of $\mathrm{C}$. jejuni was suppressed by the infection of phage CP39 until $18 \mathrm{~h}$. After $18 \mathrm{~h}, \mathrm{C}$. jejuni started to proliferate, indicating that the phage resistance subpopulation already occurred during co-cultivation. After $36 \mathrm{~h}$, the antimicrobial effect of CP 39 was eliminated, while the phage-treated group entered the exponential growth phase with the $\mathrm{OD}_{600}$ close to control group (Figure $4 \mathrm{~A}$ ). At each time point, the concentration of CP39 was evaluated by the plaque assay. The results demonstrated that after $12 \mathrm{~h}$ the phage growth remained in stationary phase (Figure $4 \mathrm{~A}$ ).

After 36 h co-cultivation of phage CP39 and C. jejuni NCTC12662, the mixture was serially diluted on the CCDA plate, and 100 colonies were randomly selected for PCR sequencing of 06875 . Nine mutation sites were detected in the 06875 gene, with a mutation frequency of 32 (Figure 4B). The most frequent mutation $(n=17)$ was at the 492/1050 bp, in which the deletion of T occurred. Besides the most frequent mutation site, the deletion or insertion of $\mathrm{T}$ also occurred at 599/1050 bp $(n=6), 977 / 1050 \mathrm{bp}(n=3)$, and $189 / 1050$ bp $(n=1)$ (Figure 4B). Other mutations included the deletion of a short sequence between 345 and 350/1050 bp and the deletion of one adenine (A) at 718/1050 bp, the replacement of $A$ to $G$ at $84 / 1050 \mathrm{bp}(n=1)$, cytosine $(C)$ to $G$ at $752 / 1050 \mathrm{bp}(n=1)$, and $\mathrm{G}$ to $\mathrm{T}$ at $813 / 1050 \mathrm{bp}(n=1)$ (Figure $4 \mathrm{~B})$. To confirm the occurrence of phage resistance in C. jejuni NCTC 12662, we further constructed a T-deletion mutant at 492/1050 bp in 06875, which was the highest mutation frequency according to the PCR sequence result. No phage plaques were observed on bacterial lawns of 06875-492delT point mutant after phage CP39 infection (Figure S2B). The above result demonstrated that the high mutation frequency of 06875 during phage CP39 infection plays an essential role in the evolution of a phage-resistant subpopulation. 
A

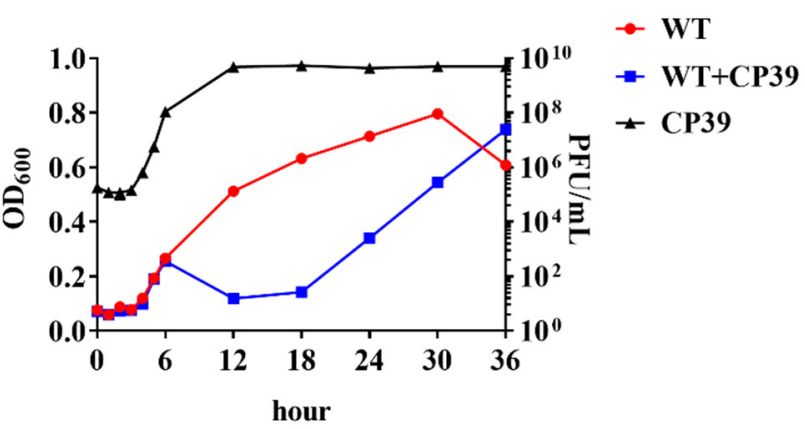

B

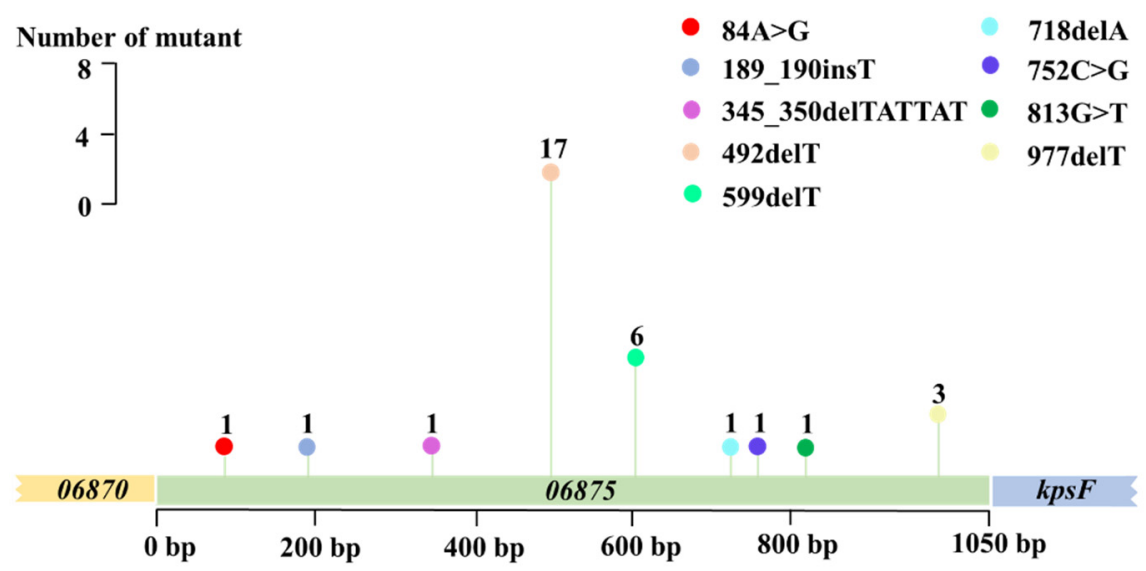

Figure 4. The occurrence of phage resistance in C. jejuni NCTC12662 and the mutation frequency of the 06875 gene during CP39 infection. (A) Generation of phage lysis curves and phage-resistant bacterial outgrowth. NCTC12662 was infected with CP39 for $36 \mathrm{~h}$ at an MOI of 0.01. NCTC12662 without phage was served as the wild type (WT) control. The one-step growth curve of phage CP39 was evaluated by the PFU $/ \mathrm{mL}$ at each time point. (B) The mutation frequency of the 06875 gene. The mutation frequency was calculated as the number of 06875 mutant from 100 phage-resistant colonies by PCR sequencing. A " $>$ " represented the original base pair was replaced by another base pair; A "_" represented an insertion (ins) or deletion (del) was occurred between base pairs.

\section{Discussion}

The application of bacteriophages to reduce the contamination of foodborne pathogens has been a hotspot in recent years. However, the frequent occurrence of phage resistance has prevented the application of phage as antibacterial agents for feed and food production [20-22]. We characterized phage CP39 and investigated the development of phage resistance in C. jejuni during CP39 infection. The phage morphology and the WGS analysis demonstrated that phage CP39 was closely related to the $C$. jejuni phage Los 1 (Figure 1B,C). Both phage CP39 and Los1 belonged to the family Myoviridae with a similar genome size of $130,715 \mathrm{bp}$ and $134,073 \mathrm{bp}$, respectively, indicating that phage CP39 belonged to Group III phage of the Cp8unalikevirus [17,23,24]. The phylogenetic analysis of CP39 from other studies has also proven our results in that CP39 was cluttered to the group III Campylobacter phage $[25,26]$. The group III phage was known to exhibit a strong lytic activity, and its successful application could effectively decrease the contamination load C. jejuni in chickens [27].

The most common strategy for bacteria to defend phages is the modification of the phage receptors. We primarily determined the receptor of CP39 according to a previous study that grouped Campylobacter phages to the CPS-dependent or motile flagelladependent phages [7]. Phage CP39 was primarily confirmed as CPS-dependent phage by the adsorption of phage in the wild type NCTC12662 and $\Delta m o t A$ mutant, but not the $\Delta k p s M$ mutant [28]. It is known that the phage-host interaction occurs beyond the cell surface. A 
recent study demonstrated that the group III phage NCTC 12673 required flagellar motility for the infection but did not need the flagella for the phage adsorption to the cell surface. Based on the previous studies, we constructed a $\triangle$ fla $A B$ mutant lacking flagellar filaments, which was sensitive to the phage infection, indicating that the motility or flagellum was not necessary for phage CP39 to infect bacteria [29].

The phase variation of the phage receptors helps the bacteria to resist against phages in a reversible manner, which could preserve the biological function of the population and develop the phage resistant sub-population for survival $[12,30]$. MeOPN encoding by the 06810 gene has been known as a receptor of NCTC12662 for several CPS-dependent phages [12]. The switch in the length of poly $G$ tract in the 06810 gene results in the expression of a non-functional protein of MeOPN and allowed the C. jejuni to resist against the phage [12]. A similar phenomenon was also observed in NCTC 11168, where poly G regulated phase variation occurred in both the cj1421 gene encoding MeOPN transferase and the cj1426 gene encoding the 6-O-Me transferase to defend the CPS-dependent phage infection [19]. We identified the genes relating to the adsorption of phage CP39 by SNP analysis, and one isolate, 12662_CP39R3, contained a deletion of T in the 06875 gene encoding CGPTase in CPS loci. The deletion of T was in a poly T tract $(8 \times \mathrm{Ts})$ and caused the truncated expression of 06875 and influenced the adsorption of phage CP39. CGPTase is known to be involved in the synthesis of the wall teichoic acid (WTA) by transferring the glycerol-phosphate units from CDP-gro to an acceptor and forming polyglycerol-phosphate chains attached to the linkage unit lipid [31,32]. The phase variable expression of 06875 may cause the lack of CDP-glycerol units during the WTA synthesis, leading to the resistance to phage CP39. The phage resistance of the $\Delta 06875$ mutant and the recovery of sensitivity in its complement strain confirmed that the phage CP39 required the CGPTase modified polyglycerol-phosphate chains for phage adsorption.

A mutation frequency of $32 \%$ was observed in the 06875 gene during the phage CP39 infection, indicating that the pressure of a specific phage would allow a rapid phase variation due to the frame shifting, and developed the phage-resistant subpopulation in C. jejuni. In addition, mutations randomly occurred in different sites of the 06875 gene, including the point mutation or replacement of a short sequence, but no mutations were located in homopolymeric tracts as with previously reported genes encoding $\mathrm{MeOPN}$ transferase [12,19]. Besides the 06875 gene, several SNPs were also observed in genes, including 03815, 06335, 06385, 06420, 06565, and 06875. Both 06335 and 06385 encode flagellate receptor-transporter protein PseD. The down-regulated expression of PseD could prevent the infection of a flagellotropic phage FLaGrab [33]. However, we did not observe any evidence that the infection of phage CP39 required flagella, as both $\triangle m o t A$ and $\triangle$ fla $A B$ were sensitive to phage CP39. The rapid development of phage resistance and the complex defense mechanisms indicated that it is essential to understand the phage-host interaction before applying phages in food production.

\section{Conclusions}

Our study demonstrated that phage CP39 was a CPS-dependent phage belonging to the Myoviridae family. Phage adsorption required the 06875 gene encoding the CGPTase in C. jejuni NCTC12662. During the phage infection, a high mutation frequency of 06875 with random mutation sites would cause the rapid development of phage resistance by the phase variable expression of the non-functional protein. Therefore, we proposed that preventing or inhibiting phage CP39 mainly depends on the phase variable gene expression of 06875, while the occurrence of SNPs in genes except for CPS loci also indicated a diversity mechanism of phage-host interaction. Our study provides additional information concerning the evolutionary adaption of $C$. jejuni against phages, which would be necessary before phage application in the industry.

Supplementary Materials: The following supporting information can be downloaded at: https: / / www.mdpi.com/article/10.3390/v14030485/s1, Figure S1: Growth curves of $\Delta m o t A$ and $\Delta$ kpsM 
mutant strain. The mot $A$ and $k p s M$ mutant strain incubated in $\mathrm{MH}$ broth for $24 \mathrm{~h}$. The same culture NCTC12662 served as the control; Figure S2: Sensitivity assay of mutant strains to CP39. (A) Sensitivity of 06875_492delT mutant strain to CP39. (B) Sensitivity of $\triangle$ flaAB to CP39; Table S1: Strains and plasmids used in this study; Table S2: Referece strains for the phylogenetic analysis; Table S3: Primers used in this study; Table S4: C. jejuni NCTC12662 genes with SNPs after phage CP39 infection.

Author Contributions: Conceptualization, Y.T., J.L. and J.H.; methodology, Y.T., J.L., Y.W. and H.Y.; software, Z.S.; validation, Y.T., J.L. and Z.S.; formal analysis, Y.T.; writing-original draft preparation, J.L.; writing—review and editing, Y.T.; visualization, Y.T.; supervision, X.J. and J.H.; project administration, Y.T. and J.H.; funding acquisition, J.H and L.K. All authors have read and agreed to the published version of the manuscript.

Funding: This study was supported by the National Natural Science Foundation of China (Grant No. 31872493) and Taishan Industry Leading Talents Project in Shandong Province (Grant No. tscy20190113).

Institutional Review Board Statement: Not applicable.

Informed Consent Statement: Not applicable.

Data Availability Statement: Publicly available datasets were analyzed in this study. The phage CP39 genome information can be found with accession number MH107028 in ENA database; The phage CP39 resistant isolates including 12662_CP39R1, 12662_CP39R2, 12662_CP39R3, 12662_CP39R4 can be found with accession number PRJNA798540 in NCBI database.

Conflicts of Interest: The authors declare no conflict of interest.

\section{References}

1. Weitz, J.S.; Poisot, T.; Meyer, J.R.; Flores, C.O.; Valverde, S.; Sullivan, M.B.; Hochberg, M.E. Phage-bacteria infection networks. Trends Microbiol. 2013, 21, 82-91. [CrossRef] [PubMed]

2. Labrie, S.J.; Samson, J.E.; Moineau, S. Bacteriophage resistance mechanisms. Nat. Rev. Microbiol. 2010, 8, 317-327. [CrossRef] [PubMed]

3. Bertozzi Silva, J.; Storms, Z.; Sauvageau, D. Host receptors for bacteriophage adsorption. FEMS Microbiol. Lett. 2016, 363 , fnw002. [CrossRef]

4. Epps, S.V.; Harvey, R.B.; Hume, M.E.; Phillips, T.D.; Anderson, R.C.; Nisbet, D.J. Foodborne Campylobacter: Infections, metabolism, pathogenesis and reservoirs. Int. J. Environ. Res. Public Health 2013, 10, 6292-6304. [CrossRef] [PubMed]

5. Rasschaert, G.; De Zutter, L.; Herman, L.; Heyndrickx, M. Campylobacter contamination of broilers: The role of transport and slaughterhouse. Int. J. Food Microbiol. 2020, 322, 108564. [CrossRef] [PubMed]

6. Zhou, J.; Zhang, M.; Yang, W.; Fang, Y.; Wang, G.; Hou, F. A seventeen-year observation of the antimicrobial susceptibility of clinical Campylobacter jejuni and the molecular mechanisms of erythromycin-resistant isolates in Beijing, China. Int. J. Infect. Dis. 2016, 42, 28-33. [CrossRef]

7. Sørensen, M.C.; Gencay, Y.E.; Birk, T.; Baldvinsson, S.B.; Jackel, C.; Hammerl, J.A.; Vegge, C.S.; Neve, H.; Brøndsted, L. Primary isolation strain determines both phage type and receptors recognised by Campylobacter jejuni bacteriophages. PLoS ONE 2015, 10, e0116287. [CrossRef] [PubMed]

8. Liang, H.; Zhang, A.; Gu, Y.; You, Y.; Zhang, J.; Zhang, M. Genetic Characteristics and multiple-PCR development for capsular identification of specific serotypes of Campylobacter jejuni. PLoS ONE 2016, 11, e0165159. [CrossRef]

9. Guerry, P.; Poly, F.; Riddle, M.; Maue, A.C.; Chen, Y.H.; Monteiro, M.A. Campylobacter polysaccharide capsules: Virulence and vaccines. Front. Cell. Infect. Microbiol. 2012, 2, 7. [CrossRef]

10. McNally, D.J.; Jarrell, H.C.; Li, J.; Khieu, N.H.; Vinogradov, E.; Szymanski, C.M.; Brisson, J.R. The HS:1 serostrain of Campylobacter jejuni has a complex teichoic acid-like capsular polysaccharide with nonstoichiometric fructofuranose branches and O-methyl phosphoramidate groups. FEBS J. 2005, 272, 4407-4422. [CrossRef]

11. Sørensen, M.C.; van Alphen, L.B.; Harboe, A.; Li, J.; Christensen, B.B.; Szymanski, C.M.; Brøndsted, L. Bacteriophage F336 recognizes the capsular phosphoramidate modification of Campylobacter jejuni NCTC11168. J. Bacteriol. 2011, 193, 6742-6749. [CrossRef] [PubMed]

12. Gencay, Y.E.; Sorensen, M.C.H.; Wenzel, C.Q.; Szymanski, C.M.; Brondsted, L. Phase variable expression of a single phage receptor in Campylobacter jejuni NCTC12662 influences sensitivity toward several diverse CPS-dependent phages. Front. Microbiol. 2018, 9, 82. [CrossRef]

13. Frost, J.A.; Kramer, J.M.; Gillanders, S.A. Phage typing of Campylobacter jejuni and Campylobacter coli and its use as an adjunct to serotyping. Epidemiol. Infect. 1999, 123, 47-55. [CrossRef] [PubMed] 
14. Li, X.; Chai, Q.; Zheng, L.; Huang, P.; Gundogdu, O.; Jiao, X.; Tang, Y.; Huang, J. Investigating the role of BN-domains of FlhF involved in flagellar synthesis in Campylobacter jejuni. Microbiol. Res. 2021, 256, 126944. [CrossRef]

15. Miller, W.G.; Bates, A.H.; Horn, S.T.; Brandl, M.T.; Wachtel, M.R.; Mandrell, R.E. Detection on surfaces and in Caco-2 cells of Campylobacter jejuni cells transformed with new $g f p$, yfp, and $c f p$ marker plasmids. Appl. Environ. Microbiol. 2000, 66, 5426-5436. [CrossRef] [PubMed]

16. Scott, A.E.; Timms, A.R.; Connerton, P.L.; Loc Carrillo, C.; Adzfa Radzum, K.; Connerton, I.F. Genome dynamics of Campylobacter jejuni in response to bacteriophage predation. PLoS Pathog. 2007, 3, e119. [CrossRef]

17. O'Sullivan, L.; Lucid, A.; Neve, H.; Franz, C.; Bolton, D.; McAuliffe, O.; Paul Ross, R.; Coffey, A. Comparative genomics of Cp8viruses with special reference to Campylobacter phage vB_CjeM_los1, isolated from a slaughterhouse in Ireland. Arch. Virol. 2018, 163, 2139-2154. [CrossRef]

18. Aidley, J.; Sorensen, M.C.H.; Bayliss, C.D.; Brondsted, L. Phage exposure causes dynamic shifts in the expression states of specific phase-variable genes of Campylobacter jejuni. Microbiology 2017, 163, 911-919. [CrossRef]

19. Holst Sorensen, M.C.; van Alphen, L.B.; Fodor, C.; Crowley, S.M.; Christensen, B.B.; Szymanski, C.M.; Brondsted, L. Phase variable expression of capsular polysaccharide modifications allows Campylobacter jejuni to avoid bacteriophage infection in chickens. Front. Cell. Infect. Microbiol. 2012, 2, 11.

20. Kortright, K.E.; Chan, B.K.; Koff, J.L.; Turner, P.E. Phage Therapy: A Renewed Approach to combat antibiotic-resistant bacteria Cell Host Microbe 2019, 25, 219-232. [CrossRef]

21. North, O.I.; Brown, E.D. Phage-antibiotic combinations: A promising approach to constrain resistance evolution in bacteria. Ann. N. Y. Acad. Sci. 2021, 1496, 23-34. [CrossRef] [PubMed]

22. Markwitz, P.; Olszak, T.; Gula, G.; Kowalska, M.; Arabski, M.; Drulis-Kawa, Z. Emerging phage resistance in Pseudomonas aeruginosa PAO1 is accompanied by an enhanced heterogeneity and reduced virulence. Viruses 2021, 13, 1332. [CrossRef] [PubMed]

23. Sails, A.D.; Wareing, D.R.; Bolton, F.J.; Fox, A.J.; Curry, A. Characterisation of 16 Campylobacter jejuni and C. coli typing bacteriophages. J. Med. Microbiol. 1998, 47, 123-128. [CrossRef]

24. Javed, M.A.; Ackermann, H.W.; Azeredo, J.; Carvalho, C.M.; Connerton, I.; Evoy, S.; Hammerl, J.A.; Hertwig, S.; Lavigne, R.; Singh, A.; et al. A suggested classification for two groups of Campylobacter myoviruses. Arch. Virol. 2014, 159, 181-190. [CrossRef] [PubMed]

25. Hooton, S.; D'Angelantonio, D.; Hu, Y.; Connerton, P.L.; Aprea, G.; Connerton, I.F. Campylobacter bacteriophage DA10: An excised temperate bacteriophage targeted by CRISPR-cas. BMC Genom. 2020, 21, 400. [CrossRef]

26. Adiguzel, M.C.; Goulart, D.B.; Wu, Z.; Pang, J.; Cengiz, S.; Zhang, Q.; Sahin, O. Distribution of CRISPR types in fluoroquinoloneresistant Campylobacter jejuni isolates. Pathogens 2021, 10, 345. [CrossRef] [PubMed]

27. Hammerl, J.A.; Jackel, C.; Alter, T.; Janzcyk, P.; Stingl, K.; Knuver, M.T.; Hertwig, S. Reduction of Campylobacter jejuni in broiler chicken by successive application of group II and group III phages. PLoS ONE 2014, 9, e114785. [CrossRef]

28. Coward, C.; Grant, A.J.; Swift, C.; Philp, J.; Towler, R.; Heydarian, M.; Frost, J.A.; Maskell, D.J. Phase-variable surface structures are required for infection of Campylobacter jejuni by bacteriophages. Appl. Environ. Microbiol. 2006, 72, 4638-4647. [CrossRef]

29. Schmidt, A.M.; Escher, U.; Mousavi, S.; Tegtmeyer, N.; Boehm, M.; Backert, S.; Bereswill, S.; Heimesaat, M.M. Immunopathological properties of the Campylobacter jejuni flagellins and the adhesin CadF as assessed in a clinical murine infection model. Gut Pathog. 2019, 11, 24. [CrossRef]

30. Sandhu, S.K.; Bayliss, C.D.; Morozov, A.Y. How does feedback from phage infections influence the evolution of phase variation in Campylobacter? PLOS Computational. Biol. 2021, 17, e1009067. [CrossRef]

31. Pooley, H.M.; Abellan, F.X.; Karamata, D. CDP-glycerol:poly(glycerophosphate) glycerophosphotransferase, which is involved in the synthesis of the major wall teichoic acid in Bacillus subtilis 168, is encoded by tagF (rodC). J. Bacteriol. 1992, 174, 646-649. [CrossRef] [PubMed]

32. Fitzgerald, S.N.; Foster, T.J. Molecular analysis of the tagF gene, encoding CDP-Glycerol:Poly(glycerophosphate) glycerophosphotransferase of Staphylococcus epidermidis ATCC 14990. J. Bacteriol. 2000, 182, 1046-1052. [CrossRef] [PubMed]

33. Sacher, J.C.; Shajahan, A.; Butcher, J.; Patry, R.T.; Flint, A.; Hendrixson, D.R.; Stintzi, A.; Azadi, P.; Szymanski, C.M. Binding of phage-encoded FlaGrab to motile Campylobacter jejuni flagella inhibits growth, downregulates energy metabolism, and requires specific flagellar glycans. Front. Microbiol. 2020, 11, 397. [CrossRef] [PubMed] 